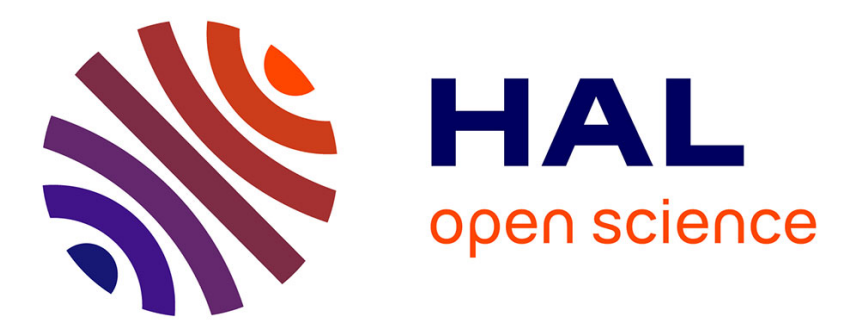

\title{
Constructions, constructionnalisation et changement linguistique.
}

Anne Carlier, Sophie Prévost

\section{To cite this version:}

Anne Carlier, Sophie Prévost. Constructions, constructionnalisation et changement linguistique.. Langue française, 2021, Nº 209 (1), pp.9-22. 10.3917/lf.209.0009 hal-03506461

\section{HAL Id: hal-03506461 https://hal.science/hal-03506461}

Submitted on 2 Jan 2022

HAL is a multi-disciplinary open access archive for the deposit and dissemination of scientific research documents, whether they are published or not. The documents may come from teaching and research institutions in France or abroad, or from public or private research centers.
L'archive ouverte pluridisciplinaire HAL, est destinée au dépôt et à la diffusion de documents scientifiques de niveau recherche, publiés ou non, émanant des établissements d'enseignement et de recherche français ou étrangers, des laboratoires publics ou privés. 


\section{Constructions, constructionnalisation et changement linguistique}

\section{Construction, constructionnalization and linguistic change}

Anne Carlier

Sorbonne Université, EA STIH

Sophie Prévost

CNRS-UMR Lattice, Universités PSL-ENS et Paris Sorbonne Nouvelle

\section{LE CADRE THEORIQUE : ETAT DE L'ART}

Les contributions qui composent ce numéro s'inscrivent dans le cadre de la grammaire des constructions. Ce courant théorique a émergé aux Etats-Unis, en réponse à des difficultés qui résultent d'une séparation stricte du lexique et de la syntaxe, notamment dans l'analyse des expressions idiomatiques (Fillmore, Kay \& O’Connor, 1988 ; Nunberg, Sag \& Wasow 1994). Comme le rappelle M. Hilpert (2014), la séparation entre lexique et syntaxe, contre laquelle s'inscrit la grammaire des constructions, est notamment ancrée dans le modèle «Dictionnaire \& Grammaire » comme conceptualisation populaire du savoir langagier : apprendre une langue reviendrait à acquérir le lexique de cette langue et les règles grammaticales qui permettent d'agencer les unités lexicales dans des propositions et des phrases. Cette séparation est par ailleurs au cœur de toute approche théorique qui considère que la seule composante du langage où se manifeste l'arbitraire du signe est le lexique, alors que la syntaxe relève des principes généraux, constitutifs du langage humain (cf. Croft \& Cruse 2004). C'est notamment le cas dans l'approche « Principes et Paramètres » de la grammaire générative (Chomsky 1981, 1992), qui admet l'existence d'une Grammaire universelle, ayant un caractère inné, dont les grammaires des langues particulières sont des instanciations résultant de l'ajustement de certains paramètres (Goldberg 1995 : 1).

Cette approche théorique de la grammaire des constructions a connu durant les deux dernières décennies un succès grandissant auprès des linguistes, en touchant également les domaines de la psycho- et neurolinguistique. Elle fait désormais partie des paradigmes théoriques classiques en s'étant dotée de ses propres revues (Constructions et Constructions and Frames), d'un colloque biennal (International Conference on Construction Grammar) et d'ouvrages de référence, dont The Oxford Handbook of Construction Grammar, édité par T. Hoffmann et G. Trousdale en 2013, ainsi qu'une introduction à la théorie et son application à l'anglais réalisée par M. Hilpert (2014), visant également un public d'étudiants. La grammaire des constructions a trouvé aussi un ancrage dans le domaine de la linguistique française, 
les premières études réalisées outre-atlantique (Michaelis \& Lambrecht 1996, Achard 1998) étant suivies par des travaux se donnant pour objectif de diffuser le cadre théorique en France (François \& Legallois 2006 ; François 2008). On mentionnera en particulier le récent numéro de Langue française intitulé «Les constructions comme unités de la langue : illustrations, évaluation, critique » $\left(\mathrm{n}^{\circ} 194\right.$, 2017). Alors que les études réunies dans ce numéro portent sur le français moderne, les contributions du présent numéro explorent comment le cadre théorique de la Grammaire des constructions peut être mis à profit pour l'analyse des changements constructionnels, voire de l'émergence de nouvelles constructions au cours de l'histoire du français.

Dans la suite de l'introduction, qui n'a d'autre ambition que d'éclairer l'orientation théorique des contributions à ce recueil, nous définirons d'abord la notion de « construction » dans le sens technique qu'elle a dans ce cadre théorique ( $§ 1)$, nous mettrons ensuite en évidence comment les constructions en tant qu'éléments de base du système langagier sont organisées dans un ensemble structuré hiérarchiquement (§ 2), et nous soulignerons enfin l'importance de l'observation empirique de l'usage, en particulier quand il s'agit de mobiliser ce cadre théorique pour rendre compte du changement linguistique $(\S 3)$. La dernière section $(\S 4)$ présentera les différentes contributions du recueil.

\section{LA NOTION DE CONSTRUCTION}

Comme l'explique le philosophe des sciences Thomas S. Kuhn (1970), en s'inscrivant à l'encontre d'une conception purement cumulative de la progression de la science telle qu'elle a été soutenue par Karl Popper (1935), il arrive qu'un paradigme scientifique, confronté à des faits récalcitrants qui le mettent en défaut, réagisse non pas en se mettant en cause, mais plutôt en qualifiant ces données non expliquées de marginales ou atypiques. Tel a été le traitement réservé aux expressions idiomatiques : reléguées dans les annexes des dictionnaires, elles ont été considérées comme des expressions lexicales complexes de nature non compositionnelle, c'est-dire dont le sens ne se laisse pas décoder à partir de celui de leurs éléments constitutifs.

Deux articles majeurs, publiés dans Language, ont mis à l'avant-plan les expressions idiomatiques et questionné leur rôle par rapport à l'approche générative. Nunberg, Sag \& Wasow (1994) montrent que l'idiomaticité est à tort identifiée à la non-compositionnalité alors que la caractéristique globale des expressions idiomatiques est plutôt leur caractère conventionnel, reposant sur une association arbitraire entre forme et sens. Si certaines expressions idiomatiques sont réellement non compositionnelles (par ex. se casser la pipe 'mourir', prendre la mouche 'se fâcher'), pour d'autres le sens est bel et bien distribué sur les éléments constitutifs (tirer profit, tirer les ficelles, avec tirer 'exploiter' et les ficelles 'les connexions personnelles'), ce qui explique qu'elles peuvent avoir une certaine flexibilité syntaxique. Fillmore, Kay \& O'Connor (1988) adoptent une approche plus radicale : l'idiomaticité, loin d'être un fonctionnement irrégulier de la langue, bat en brèche la séparation entre unités du lexique, stockées dans la mémoire, et les règles de la grammaire permettant d'assembler les unités lexicales dans des constructions complexes, et conduit à repenser la relation entre lexique et syntaxe en termes de continuum, car 
elle oblige à admettre l'existence d'unités de langue qui sont composées de plusieurs unités lexicales. Dans cette nouvelle approche, l'unité de la description devient la construction, celle-ci pouvant se situer à différents degrés de complexité et varier quant à sa taille. Le tableau suivant, qui s'appuie sur Goldberg (2006 : 5), en offre des exemples :

Tableau $n^{\circ} 1$ : Exemples de constructions, variant en taille et en complexité

\begin{tabular}{|l|l|}
\hline Morphème & pré-, -eur \\
\hline Mot & livre, mais \\
\hline Mot construit & V-N, ex. porte-clé \\
\hline Expression idiomatique lexicalement saturée & Les carottes sont cuites ! \\
\hline $\begin{array}{l}\text { Expression idiomatique ayant des positions } \\
\text { structurales ouvertes }\end{array}$ & X donne du fil à retordre à $Y$ \\
\hline Construction corrélative & Plus il réfléchit, moins il comprend \\
\hline Construction ditransitive & $\begin{array}{l}\mathrm{S} \vee \\
\text { doux OD Ol ex. Pierre lui a glissé un mot }\end{array}$ \\
\hline Passif & $\begin{array}{l}\mathrm{S} \text { être } \\
\text { tous }\end{array}$ \\
\hline
\end{tabular}

Est considérée comme construction une unité qui associe d'une manière conventionnelle une forme et un sens et dont certains aspects formels ou sémantiques ne sont pas strictement prévisibles à partir des éléments constitutifs de cette construction ou d'une autre construction identifiée. Cette définition s'applique à l'évidence aux unités de base que sont les morphèmes, mais également aux syntagmes complexes dont soit la forme soit le sens ne peut pas être (entièrement) dérivé à partir des unités qui les composent et à partir des constructions existant par ailleurs dans la langue. De plus, peuvent être stockées comme constructions des séquences qui, tout en étant prévisibles, sont extrêmement fréquentes, telles que je t'aime, excusez-moi.

Parmi les différents types d'expressions idiomatiques établies par Fillmore \& al. (1988), nous retiendrons la distinction entre expressions idiomatiques dites «substantives » ou lexicalement instanciées, et expressions idiomatiques « formelles » ou schématiques : dans le premier cas, tous les éléments de l'expression sont figés (par ex. les carottes sont cuites), alors que dans le second cas, certaines positions sont ouvertes et peuvent être instanciées par une classe d'expressions qui conviennent syntaxiquement et sémantiquement à cette position. Il est possible de distinguer des constructions ayant différents degrés de schématicité, mettant en évidence le continuum entre lexique et syntaxe :

1. a. $\mathrm{S}$ donner du fil à retordre $\mathrm{OI}_{\mathrm{à}}$

b. $\mathrm{S}$ donner OD OI

c. S V OD OI

Pierre donne du fil à retordre à sa mère.

Pierre donne un cadeau à sa mère.

Pierre lui glisse un billet de 5 euros.

Il est important de considérer que chacun de ces niveaux de schématicité conduit à une association entre forme et sens. Même le niveau le plus abstrait, exemplifié par (1c), est associé non seulement à une forme, consistant en une structure syntaxique, mais également à un sens, dans la mesure où cette construction confère au verbe glisser le trait sémantique de donation, trait dont ce verbe est dépourvu hors de cette construction (par ex. Pierre glisse sur la neige, Pierre glisse une lettre sous la porte, ...). 


\section{DU LEX-IQUE AU « CONSTRUCT-IQUE »}

La ligne de démarcation entre le lexique, contenant la connaissance des mots, d'une part, et la syntaxe, contenant la connaissance des règles permettant d'assembler les unités lexicales dans des constructions complexes, d'autre part, étant floue, la grammaire des constructions propose de l'abandonner. Elle conceptualise au contraire le savoir linguistique comme un vaste inventaire de constructions, qui par analogie avec le lex-ique stocké dans la mémoire, est appelé «construct-ique ». Cet inventaire se présente comme un réseau taxinomique de constructions où chaque construction correspond à un nœud (Croft \& Cruse 2004 : 262). Ce réseau est structuré hiérarchiquement : certaines constructions sont plus basiques ou plus générales que d'autres et les constructions de niveau inférieur héritent des propriétés de niveau supérieur (Goldberg 1995 : 72 sqq.).

Ainsi l'expression idiomatique prendre le large 'partir', constitue une construction, car son sens n'est pas prévisible à partir de ses éléments constitutifs. Cette construction instancie en même temps la structure argumentale caractéristique du verbe prendre, qui est celui d'un verbe transitif, et va ainsi hériter des propriétés des constructions plus schématiques dont elle relève. C'est ainsi que le savoir linguistique, ayant trait à la construction prendre le large se trouve stocké à différents niveaux d'abstraction. Les différents niveaux de schématicité, ainsi que les liens d'héritage, sont représentés dans (2).

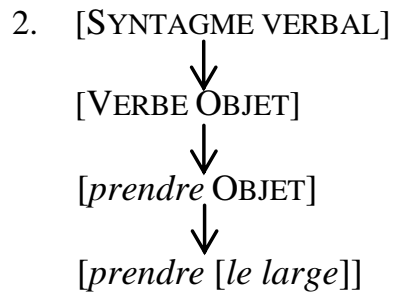

La construction prendre le large s'insère ainsi dans un réseau de constructions organisé hiérarchiquement :

Figure $n^{\circ} 1:$ Réseau des constructions

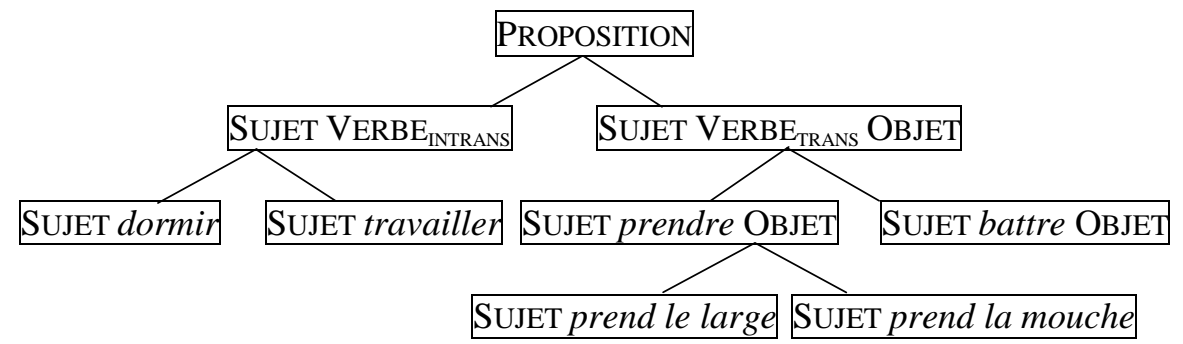

Comme le fait remarquer Hilpert (2014: chap. 3), les niveaux plus concrets peuvent hériter de caractéristiques constructionnelles, formelles ou sémantiques, à 
partir des niveaux plus schématiques, l'inverse n'étant pas vrai. Ainsi, le français possède une construction prépositionnelle locative qui fait intervenir nécessairement l'article défini et qui a parfois été analysée comme pseudo-générique, dans la mesure où elle fait référence à un type de lieu plutôt que de spécifier un lieu ayant des propriétés particulières.

\section{3. a. Pierre passe ses vacances à la mer. \\ b. Pierre ne va plus à l'école. \\ c. Pierre est sorti de l'hôpital. \\ d. Pierre va au lit.}

Cette construction hérite à partir de la construction du syntagme prépositionnel d'un certain nombre de contraintes formelles générales, par exemple le fait que la préposition précède le syntagme nominal défini. Mais elle possède aussi des propriétés spécifiques qui ne sont pas partagées par toutes les instances possibles de la construction prépositionnelle. Ces propriétés sont formelles et sémantiques. Côté formel, on notera par exemple que l'article défini n'alterne pas avec d'autres déterminants. Du point de vue sémantique, ces constructions sont nécessairement liées à un scénario stéréotypique, ce qui contraint la nature du sujet : dans la phrase (3b) le sujet fait référence à un élève et non pas à un professeur, alors que dans $(3 \mathrm{c})$ le sujet réfère à un patient plutôt qu'à un docteur; de plus, le choix du nom inclus dans le syntagme prépositionnel n'est pas libre, comme le montre ?Pierre va au sofa, formé à partir de $(3 \mathrm{~d})$. Ces contraintes spécifiques à la construction exemplifiée par les exemples dans (3) ne sont à l'évidence pas partagées par les syntagmes prépositionnels en général et ne sont donc pas projetées vers les niveaux plus schématiques.

Comme montré ci-dessus, les liens d'héritage peuvent communiquer des propriétés non seulement formelles, mais également sémantiques. Les exemples (4a-b) offrent un autre exemple illustrant la nature sémantique du lien d'héritage : la construction [SUJET être d'un ADJECTIF] conduit nécessairement à interpréter l'adjectif comme évoquant le haut degré d'une propriété scalaire.

4. a. Ce brie est d'un onctueux! (R. Sabatier, Les filles chantantes)

b. Certaines de mes compââânes étaient d'un grossier! (Albertine Sarrazin, La Cavale)

Selon Goldberg (1995: 74-81), quatre types de liens d'héritage peuvent être distingués : les liens de polysémie, les liens de sous-partie, le lien d'instanciation et les liens d'extension métaphorique.

Il est question d'un lien d'instanciation, qui constitue le lien d'héritage de base, quand une construction est une version plus spécifique d'une autre construction et hérite donc des propriétés syntaxiques et sémantiques de cette construction tout en pouvant présenter des traits propres. Ainsi l'expression idiomatique prendre le large (2) entretient un lien d'instanciation avec la construction prendre suivi d'un objet dans la construction transitive.

Les liens d'héritage de type polysémique sont illustrés par les différentes acceptions de la construction ditransitive dans les exemples sous (5). 
5. a. Marie lui donne un livre.

b. Marie lui tricote un pull.

c. Le docteur lui a autorisé une sortie.

d. Marie lui a refusé l'accès.

Les différentes acceptions de la construction ditransitive sont motivées par le sens central qui est celui d'un transfert effectif, mais ne sont pas pour autant des instanciations de ce scénario de transfert. Ainsi (5b), appelé parfois construction bénéfactive, n'évoque pas un transfert à proprement parler, mais une intention de transfert, alors que $(5 \mathrm{c})$ fait référence à une possibilité de transfert, et $(5 \mathrm{~d})$ à un blocage de transfert.

Il est question d'un lien de sous-partie quand une construction se présente comme une sous-partie d'une autre construction. L'exemple donné par Goldberg (1995 : 78) est celui des verbes de mouvement transitifs et intransitifs, où la construction intransitive de mouvement se présente comme une sous-partie de la construction causative de mouvement :

6. a. Marie sort la voiture du garage.

b. La voiture sort du garage.

Figure $\mathrm{n}^{\circ}$ 2: Lien d'héritage entre constructions causative de mouvement et construction intransitive de mouvement

Construction causative de mouvement (ex. 6a)

\begin{tabular}{|c|c|c|c|c|c|}
\hline Sém & $\begin{array}{c}\text { CAUSE-MOUVEMENT } \\
\mid \\
\text { PRED } \\
\downarrow \\
\mathrm{V} \\
\end{array}$ & $<\left.\right|_{\substack{<\text { cause } \\
\downarrow}} ^{\downarrow}$ & $\begin{array}{l}\text { thème } \\
\downarrow \\
\text { OBJET }\end{array}$ & $\begin{array}{r}\text { but } \\
\downarrow \\
\text { OBJE } \\
\end{array}$ & $\begin{array}{r}> \\
> \\
\text { ECT }\end{array}$ \\
\hline \multicolumn{4}{|c|}{ Construction intransitive de mouvement (ex. 6b) } & \multicolumn{2}{|c|}{ HERITAGE SOUS-PARTIE } \\
\hline Sém & $\begin{array}{c}\text { MOUVEMENT } \\
\mid \\
\text { PRED } \\
\downarrow \\
\mathrm{V}\end{array}$ & $<\left.\right|_{\substack{<\mathrm{e} \\
\text { OBJET }}} ^{\downarrow}$ & & $\begin{array}{l}\downarrow_{\text {OBJET I }}^{\text {but }} \\
\downarrow\end{array}$ & $\begin{array}{l}> \\
>\end{array}$ \\
\hline
\end{tabular}

Un dernier type de lien d'héritage, mentionné par Goldberg (1995 : 81) et Hilpert (2014), fait intervenir la notion de métaphore. La métaphore fondamentale à l'œuvre dans l'exemple (7) consiste à conceptualiser le changement en termes de mouvement spatial. C'est ainsi que le verbe tomber, évoquant un mouvement abrupt 
et non voulu, peut aussi, en se combinant avec un adjectif attribut, évoquer un changement d'état abrupt et non voulu.

\section{Pierre tomba (malade/(raide-)mort/amoureux)}

Il est à noter que le construct-ique n'est pas organisé selon une taxinomie stricte, mais que les liens d'héritage peuvent être multiples.

On conclura cette section par une remarque importante concernant les niveaux les plus schématiques, qui comprennent les catégories grammaticales et les règles combinatoires en syntagmes. Les catégories grammaticales comme l'adjectif, le déterminant, et les règles selon lesquelles elles s'agencent en syntagmes, ne relèvent nullement d'une grammaire qui serait universelle, et donc a priori pas d'une compétence linguistique innée et faisant partie du patrimoine génétique de l'humanité, mais sont spécifiques aux langues particulières. Il a été montré empiriquement que les locuteurs gardent en mémoire de riches répertoires de formes fléchies, de fragments de phrases, etc. (Bybee 2006) et qu'ils mettent à jour leur savoir linguistique en fonction de leurs expériences du langage. Dans cette optique, le construct-ique est usage-based, c'est-à-dire créé par l'usage effectif de la langue et continuellement influencé par cet usage effectif de la langue.

\section{ChANGements CONStRuctionnels et USAGE}

La dimension usage-based de la grammaire des constructions conduit à accorder une place centrale à la variation. Une construction étant définie comme une association conventionnelle entre une forme et un sens, cette variation peut concerner la forme tout comme le sens.

- D'une part, les locuteurs savent qu'une construction peut être réalisée formellement de différentes manières, que les variations soient d'ordre phonétique, morphologique ou syntaxique. On mentionnera à titre d'exemple l'alternance entre [we] vs [wa], qui s'opposent comme étant respectivement la prononciation savante et la prononciation populaire dès le début du XIII ${ }^{\mathrm{e}}$ s. jusqu'à la veille de la Révolution française. D'autres exemples, dans le domaine de la syntaxe et de la morphosyntaxe, sont analysés dans le présent numéro: la position préverbale ou postverbale d'un sujet (S. Prévost) et l'alternance entre constructions anticausatives pronominales et non pronominales (L. Schøsler).

- D'autre part, les locuteurs savent que la construction a le potentiel de transmettre une série de significations différentes, cette variation pouvant être appréhendée en termes de polysémie. Outre l'exemple (5) mentionné ci-dessus, on peut évoquer l'exemple de la construction pronominale, qui, comme le montre D. Creissels (2006 : chap. 22), présente un emploi proprement réfléchi (8a, 'Pierre fait porter son admiration sur lui-même'), un emploi auto-causatif ( $8 b$, Pierre est agent et patient du procès, mais n'exerce pas l'activité exprimé par le verbe sur lui-même), un emploi non-causatif ( $8 \mathrm{c}$, le processus ne suggère pas l'intervention d'une cause externe identifiable ou minimise l'intervention d'une force externe en insistant sur la prédisposition du sujet à subir le procès) et un emploi proprement passif (8d, 'On a vendu ce livre a des milliers d'exemplaires'). 
8. a. Pierre s'admire.

b. Pierre se lève.

c. La porte s'ouvre. / Ce tissu se lave facilement.

d. Ce livre s'est vendu à des milliers d'exemplaires.

Etant donné cette variation qui affecte à la fois la forme et le sens des constructions, la connaissance qu'ont les locuteurs des constructions se traduit non pas comme une mise en rapport bi-univoque d'une forme et d'un sens pour chacune des constructions, mais plutôt comme une mise en rapport d'un ensemble de formes connexes et de sens connexes, associée à une compréhension fine des facteurs qui conditionnent cette variation. Les locuteurs savent en effet que telle forme est rare, voire inappropriée dans tel contexte linguistique ou dans telle situation énonciative. Ainsi trois formes se font concurrence pour l'expression du futur: le présent, le futur dit périphrastique aller + infinitif, et le futur simple ou synthétique.

9. a. Nous arrivons demain à 8 heures.

b. Nous allons arriver demain à 8 heures.

c. Nous arriverons demain à 8 heures.

Le choix entre les trois variantes morphologiques pour l'expression du futur n'est toutefois pas aléatoire, mais il est conditionné par des facteurs extralinguistiques, de nature sociolinguistique, et par des facteurs internes à la langue. Pour ce qui est des facteurs extralinguistiques, il est généralement admis que la forme périphrastique (9b) est plus familière et plus orale que la forme synthétique (9c). Pourtant, selon l'étude statistique menée par Poplack \& Dion (2009), un facteur significatif, largement ignoré dans la tradition grammaticale, est de nature interne à la langue : la présence de la négation, même dans le registre oral, déclenche l'emploi du futur synthétique, ainsi que l'illustrent les exemples (10) et (11) :

10. Dire que dans quatre cents ans d'ici bien, il va avoir encore des Fauteux puis ils vont encore parler français! Qu'ils parleront pas l'anglais.

(Ottowa-Hull corpus.004.36)

11. il va virer la clef, il va la rebarrer puis va la redébarrer. Il s'apercevra pas qu'elle était débarré (Récits du français québécois d'autrefois.02 1.1256)

C'est à partir de cette variation affectant à la fois la forme et le sens des constructions que naît le changement constructionnel : les facteurs qui conditionnent la variation étant susceptibles d'évoluer, certaines formes ou significations peuvent être privilégiées ou au contraire passer à l'arrière-plan. En guise d'exemple pour le changement formel, on reprendra l'exemple de l'alternance phonétique [we] versus [wa] mentionnée ci-dessus: la prononciation dite populaire [wa] s'impose après la Révolution française, et cesse donc d'être marquée comme populaire. Un exemple pour le changement sémantique est offert par les noms de rôle féminins qui développent un sens ayant trait aux intrigues et liaisons amoureuses hors mariage que n'ont pas leurs contreparties au masculin (maître/ maîtresse, courtisan / courtisane, entremetteur / entremetteuse), sens qui finit par dominer. Cette évolution sémantique, qui n'est d'ailleurs pas propre au français (par ex. en anglais : Madam 'femme qui gère une maison de prostitution'), s'explique par le contexte sociologique où elle a émergé, caractérisé par un rapport spécifique homme-femme. 
Il est possible de s'interroger sur ce qui déclenche une évolution des facteurs conditionnant la variation. Comme le suggèrent les deux exemples ci-dessus, des conditions extralinguistiques (les changements sociaux de la révolution française, le statut de la femme dans la société) peuvent jouer un rôle. D'autres changements dus à des conditions internes à la langue peuvent également jouer un rôle. C'est ainsi que la substitution de on à nous (ex. On arrive!) voire à vous (par ex. Alors, on s'amuse ? $)^{1}$ et le changement sémantique affectant on qui en résulte peuvent être expliqués par une stratégie d'évitement de la flexion personnelle du verbe sous forme de suffixes, qui cadre dans un changement typologique plus global caractéristique du français quant au mode d'expression de la grammaire (Carlier \& Combettes $2015: 26$ ).

Tableau $n^{\circ} 2$ : Les formes de l'indicatif présent du verbe cantare en latin et du verbe chanter en français avancé

\begin{tabular}{|c|c|c|}
\hline Indicatif présent & Latin & Français avancé \\
\hline $1 \mathrm{sg}$ & cant-o & /3-كãt/ \\
\hline $2 s g$ & canta-s & 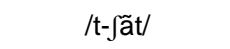 \\
\hline $3 s g$ & canta-t & /i-fãt/ \\
\hline $1 \mathrm{pl}$ & canta-mus & 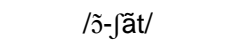 \\
\hline $2 \mathrm{pl}$ & canta-tis & 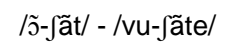 \\
\hline $3 p l$ & canta-nt & /i-fãt/ \\
\hline
\end{tabular}

Comme nous l'avons vu ci-dessus, la connaissance qu'a le locuteur des constructions implique qu'il sait qu'une construction peut être réalisée formellement de différentes façons, et peut véhiculer plusieurs sens reliés entre eux et qu'il dispose en plus d'une compréhension fine des facteurs qui conditionnent cette variation. En étudiant le changement constructionnel, nous cherchons comment le savoir linguistique qu'ont les locuteurs des constructions évolue au fil de temps. Cette étude s'appuie nécessairement sur des corpus qui permettent de reconstruire ce savoir en analysant la distribution de cette variation par rapport aux registres et par rapport au contexte linguistique, tout comme les changements de fréquence des différentes variantes en fonction de ces paramètres. Comme le souligne Bybee (2006), la montée en fréquence est un moteur important pour l'émergence de nouvelles constructions dans la mesure où elle a pour conséquence que certains aspects formels ou sémantiques ne sont plus strictement prévisibles à partir des éléments constitutifs de la construction d'origine, par des effets de réduction et fusion phonologiques et/ou par une perte de transparence sémantique (ex. par ce que > parce que $/$ pask $^{2} /$ ), ce qui conduit parfois à de nouvelles fonctions pragmatiques (ex.j'sais pas / $\int \mathrm{epa} / \mathrm{dans}$ la fonction de marqueur servant à mitiger, ainsi que tu vois, vous voyez, voyons,

\footnotetext{
1 En comparaison avec on signifiant 'nous', l'interprétation de on comme 'vous' est bien moins fréquente et est plus contrainte non seulement d'un point de vue pragmatique (possible dans un contexte où l'on interagit avec ses allocutaires), mais aussi d'un point de vue syntaxique (nous, on a décidé de partir ; *vous, on a décidé de partir?). La forme verbale de la première personne en -ons fait donc plus l'objet d'une stratégie d'évitement que la forme de la $2^{\mathrm{e}}$ personne du pluriel en -ez, homonyme pour les verbes en -er avec l'infinitif et le participe passé.
} 
voilà abordés dans la contribution de Kirsten Jeppesen Kragh dans ce recueil). Il importe néanmoins de distinguer entre la token frequency d'une construction, qui correspond au nombre total d'instances individuelles d'une construction, et sa type frequency, qui est le nombre d'instances différentes d'une construction. Une montée en fréquence peut se produire en association avec des lexèmes précis à haute fréquence (ce qui conduit à un type frequency peu élevée face à un token frequency élevée) ou peut au contraire intervenir à des niveaux schématiques (avec un ratio élevé type frequency/token frequency). Dans la lignée de Bybee (2003), nous soulignons que l'effet sur le changement linguistique n'est pas le même : alors que dans le premier cas la montée en fréquence conduit plutôt à des effets de maintien de constructions conservatrices voire à un figement lexical, dans le deuxième cas elle devient signe d'une productivité croissante et conduit à des constructions grammaticales nouvelles (Bybee 2003). C'est ce qu'illustrent la contribution de Peter Lauwers et Anaïs Vajnovszki et celle de Jasper Van Gaever dans ce numéro.

\section{LES CONTRIBUTIONS A CE RECUEIL}

Les différentes contributions à ce recueil mettent en évidence le profit qu'on peut tirer du cadre théorique de la grammaire des constructions pour étudier le changement constructionnel dans des domaines divers : la syntaxe de la phrase dans ses rapports avec la structure informationnelle, les variations quant à la structure argumentale de la construction verbale, et les marqueurs du discours.

Plusieurs études de ce recueil portent sur la question de l'ordre des constituants dans la phrase. Ainsi Peter Lauwers et Anaïs Vajnovszki se consacrent à l'antéposition de l'adjectif attribut en la considérant comme une construction qui s'articule en plusieurs sous-constructions ayant différents degrés de schématicité (comprenant des constructions ayant un faible degré de schématicité comme les constructions corrélatives et comparatives: plus le temps aura passé, plus forte sera l'impression d'infaillibilité gravée dans les mémoires (Castel 2009), voire des constructions lexicales figées : Tel est X. Rares sont les $X$ ). Ils étudient à partir de tests syntaxiques la baisse de la productivité de la construction à attribut adjectif antéposé dans son ensemble ainsi que le maintien, voire l'émergence, de diverses sous-constructions présentant ce même ordre syntaxique. Une autre étude ayant trait à l'ordre des constituants est celle de Sophie Prévost, qui traite du recul de la variabilité de la position au profit d'un ordre prévalent sujet-verbe. Adoptant une perspective constructionnelle, elle propose une approche unifiée de la position du sujet en retraçant l'émergence progressive d'une construction schématique sujetverbe (et cela quelle que soit la forme d'expression du sujet, lexicale ou pronominale), qui conduit à une spécialisation forte de la construction verbe-sujet. Cette étude associe étroitement les aspects formels (syntaxiques) et fonctionnels (sémantico-pragmatiques) dans une perspective d'association entre forme et sens, sans privilégier une dimension par rapport à l'autre.

Suivent deux études portant sur des constructions faisant intervenir le verbe de perception voir. S'attachant au latin tardif et à l'ancien français en s'appuyant sur un corpus pour chacun des deux états de langue, Jasper Vangaever montre comment, dès le latin tardif, la construction combinant le verbe voir avec un objet direct et un participe présent (On la voit attendant devant la porte) devient une construction 
autonome par rapport à la construction accusativus cum participio dont elle est issue : le verbe voir devient un introducteur d'une construction thétique à valeur aspectuelle progressive. Il avance que cette construction se maintient avec les mêmes propriétés formelles et fonctionnelles en ancien français, mettant ainsi en cause l'analyse communément répandue en termes d'attribut de l'objet. S'appuyant sur des concepts mis en avant par Hopper (1991), il fait ressortir le phénomène de «persistence » (survivance du sens de perception sensorielle de 'voir'), qui freine l'aboutissement de la constructionnalisation et conduit à un effet «layering » (coexistence de la construction source et de la construction nouvelle). La dimension aspectuelle progressive est également mise en avant dans l'étude que consacrent Kirsten Jeppesen Kragh et Lene Schøsler à la construction du verbe voir avec un objet direct combiné à une proposition relative. Les deux auteurs montrent comment cette construction est devenue, par un processus de réanalyse, un membre du paradigme aspectuel exprimant la progression.

L'article de Lene Schøsler, également consacré aux constructions verbales, fait intervenir des constructions à un niveau plus schématique. En se consacrant à l'alternance entre les constructions anticausatives non marquées et les constructions anticausatives réflexives (la branche casse, la branche se casse), l'auteure montre comment des constructions schématiques peuvent se réorganiser en de nouveaux paradigmes et participer de ce fait à la réorganisation de la grammaire. C'est ainsi qu'elle avance que les deux constructions soumises à l'étude, les constructions anticausatives réflexives et non réflexives, sont en passe de s'intégrer dans le paradigme de la voix. Le locuteur est donc amené à choisir l'un des éléments du paradigme, actif, passif, anticausatif réflexif ou anticausatif non réflexif, en fonction du contenu qu'il souhaite communiquer, ce qui conduit l'auteure à définir la distinction sémantique entre les deux types de constructions anticausatives. Une dernière contribution portant également sur la formation de paradigmes ou de sous-paradigmes, de la main de Kirsten Jeppesen Kragh, est consacrée aux marqueurs de discours formés à partir du verbe voir (tu vois, vous voyez, voyons, voilà) et savoir (tu sais/vous savez), orientés vers le locuteur et/ou l'allocutaire, et porte sur la période du $\operatorname{XIV}^{\mathrm{e}} \mathrm{s}$. au français contemporain. Son étude conduit à distinguer différentes valeurs pragmatiques, lesquelles sont initialement liées à la position occupée par les marqueurs du discours : position initiale, position insérée, position finale. Elle observe toutefois que, au cours de l'histoire, chacun des marqueurs du discours tend à se spécialiser dans une position et s'approprie ainsi, par inférence pragmatique, le trait qui y est associé. 


\section{Références bibliographiques}

ACHARD M. (1998), Representation of Cognitive Structures: Syntax and Semantics of French Sentential Complements, Berlin, Mouton de Gruyter.

BybeE J. (2006), "Mechanisms of Change in Grammaticization: The Role of Frequency", in B. D. Joseph \& R. D. Janda (éds), The Handbook of Historical Linguistics, 602-623, Oxford, Blackwell.

CARlier A. \& Combettes B. (2015), «Typologie et catégorisation morphosyntaxique : du latin au français moderne », Langue française 187(3), 15-58. [doi:10.3917/lf.187.0015].

Chomsky N. (1982). Lectures on Government and Binding: The Pisa Lectures. $2^{\text {nd }}$ Rev. ed., Dordrecht, Foris.

Coussé E, Andersson P. \& Olofsson J. (éds) (2018), Grammaticalization Meets Construction Grammar, Amsterdam, Benjamins.

CReISSELS D. (2006), Syntaxe générale: une introduction typologique, 1-2, Paris, Lavoisier.

Croft W. \& Cruse D. A. (2004), Cognitive Linguistics, Cambridge, Cambridge University Press.

FRANÇOIS J. (2008), « Les grammaires de construction un bâtiment ouvert aux quatre vents », Cahiers du CRISCO $26: 3-19$.

GisboRne N. \& PATtEN A. (2011), "Construction Grammar and grammaticalization", in H. Narrog \& B. Heine (éds), The Handbook of Grammaticalization, 92-105, Oxford, Oxford University Press.

Goldberg A. E. (1995), Constructions: A Construction Grammar Approach to Argument Structure, Chicago, University of Chicago Press.

Goldberg A. E. (2006), Constructions at Work: The Nature of Generalization in Language, Oxford, Oxford University Press.

HilPert M. (2014), Construction Grammar and its Application to English, Edinburgh, Edinburgh University Press.

Hoffmann T. \& Trousdale G. (éds) (2013), The Oxford Handbook of Construction Grammar, Oxford, Oxford University Press.

HopPeR P. J. (1991), “On some principles of grammaticization”, in E. C. Traugott \& B. Heine (éds), Approaches to Grammaticalization, Vol. I, 17-36, Amsterdam, Benjamins.

Kunn T. S. $1970^{2}$. The Structure of Scientific Revolutions, Chicago, University of Chicago Press.

Legallois D. \& Patard A. (éds) (2017), «Les constructions comme unités de la langue : Illustrations, évaluation, critique », Langue française 194.

Legallois D. \& François J. (éds) (2006), « Autour des grammaires de constructions et de patterns », Cahiers du Crisco 21.

Michaelis L. A. \& Lambrecht K. (1996), "Toward a Construction-Based Theory of Language Function: The Case of Nominal Extraposition", Language 72: 215-24.

Nunberg G., SAG I. A. \& Wasow T. (1994), "Idioms”, Language 70: 491-538.

Poplack S. \& Dion N. (2009), "Prescription vs. Praxis: The Evolution of Future Temporal Reference in French", Language 85: 557-87.

POPPER K. (1935), Logik der Forschung. Zur Erkenntnistheorie der modernen Naturwissenschaft, Vienna, Springer Verlag.

Traugott E. C. \& Trousdale G. (2010), Gradience, Gradualness and Grammaticalization, Amsterdam, Benjamins. 